\title{
Robust regression analysis of energy spectrum evolution in time for relativistic electron bursts in the Earth's magnetosphere
}

\author{
Temir Zharaspayev* \\ National Research Nuclear University MEPhI \\ E-mail: masscry@gmail.coml

\section{Sergey Aleksandrin} \\ National Research Nuclear University MEPhI \\ E-mail: syaleksandrin@mephi.ru

\section{Sergey Koldashov} \\ National Research Nuclear University MEPhI \\ E-mail: svkoldashov@mephi.ru

\section{Arkady Galper} \\ National Research Nuclear University MEPhI \\ E-mail: amgalperemephi.ru
}

\begin{abstract}
Various local geophysical phenomena, like thunderstorms and earthquakes can be the cause for particle precipitation from Earth radiation belt. Longitudinal particle drift period is known to be dependent from its energy, due to this fact: using particles energy spectrum data change in time, it is possible to determine distances between locations of precipitation and registration on board of satellite. Using results of numerical model calculations of longitudinal particle drift, the energy spectrum dynamics of particle burst electrons detected in satellite experiments ARINA (on board the Russian satellite Resurs-DK1) and VSPLESK (on board the International Space Station) have been analyzed. The robust regression method is proposed to be used for detection of burst electrons in data with high levels of background albedo electron fluxes. The research has shown that this method provides the way to increase signal to noise ratio in experimental data and achieve more precise burst precipitation area detection in compare to linear regression analysis.
\end{abstract}

The 34th International Cosmic Ray Conference,

30 July- 6 August, 2015

The Hague, The Netherlands

\footnotetext{
* Speaker.
} 


\section{Introduction}

The shape of Earth's magnetic field forms a trap for charged particles. It is called the Earth radiation belt. Some geophysical phenomena (earthquakes, thunderstorms) are known to be the cause of particle precipitations below the Earth radiation belt to heights of low orbital satellites. Precipitated particles take part in three types of motion: rotation around geomagnetic field line, bouncing between mirror points and longitudinal drift around the Earth. Satellite experiment below radiation belt with particle detection instrument crossing disturbed L-shell would detect precipitated particles as short time increase in count rate of particles. This well-known phenomenon is called burst and has been studied for long time in many satellite experiments $[1,2,3]$. If one assumes that particle precipitaion occures instantly in single point-like region(locally) and spectrum of particles defined by power law, one can expect that precipitated particles would drifts along disturbed Lshell as a cloud of particles. This article discusses determination methods of particle precipitation region based on electron burst spectrum change measurement during the burst observation time. The methods based on well-known fact that longitudinal velocity of precipitated particle depends on its energy.

\section{Particle longitudinal drift process simulation}

As it was mentioned above, longitudinal drift velocity of particles depends on their energy. The time $\left(T_{d r}\right)$ for particle to perform a complete rotation around the Earth defined by formula (1) [4]:

$$
T_{d r}=K_{1} \frac{1+\varepsilon}{\varepsilon(1+\varepsilon)} \frac{K_{2}}{L}
$$

where $\varepsilon$ is the ratio of the kinetic energy of the particle to its rest energy, $L$ - drift shell coordinate.

$$
K_{1}=\frac{4}{3} \pi \frac{Z|e|}{m_{0}} \frac{M}{c^{3}}
$$

$M$ - geomagnetic field momentum, $m_{0}$ - electron mass. The $K_{2}$ depends on latitude of particle's mirror point and varies from 1.0 to 1.5 , where $K_{2}=1.0$ on geomagnetic equator.

$$
t_{d r}(\Delta \lambda, \varepsilon)=T_{d r}(\varepsilon) \frac{\Delta \lambda}{360}
$$

where $\Delta \lambda$ - longitudinal distance between points of precipitation and registration and $\varepsilon=$ $E / E_{0}$, where $E$ is particle energy.

Using this model, one can assume that registered particles of cloud would group along the curve $t_{d r}(\Delta \lambda, \varepsilon)(2.3)$, where arrival time to detector from precipitation region depends on value of $\Delta \lambda$ and $\varepsilon$.

In this work, using Monte-Carlo method two sets of electrons were generated and followed all the way to their registration. The first set are electrons precipitated locally and instantly from radiation belt, and the second one consists of background albedo electrons. It is natural to search time of registration on particle energy dependence in form of plot where on $\mathrm{x}$-axis particle energy 
is plotted, and on y-axis its registration time. In fig. 1 an example of Monte-Carlo simulation is shown, black curve calculated using (2.3), black squares - burst electrons, gray points - background albedo electrons. Main objective of this research is to find from merged sets of background and burst electrons.
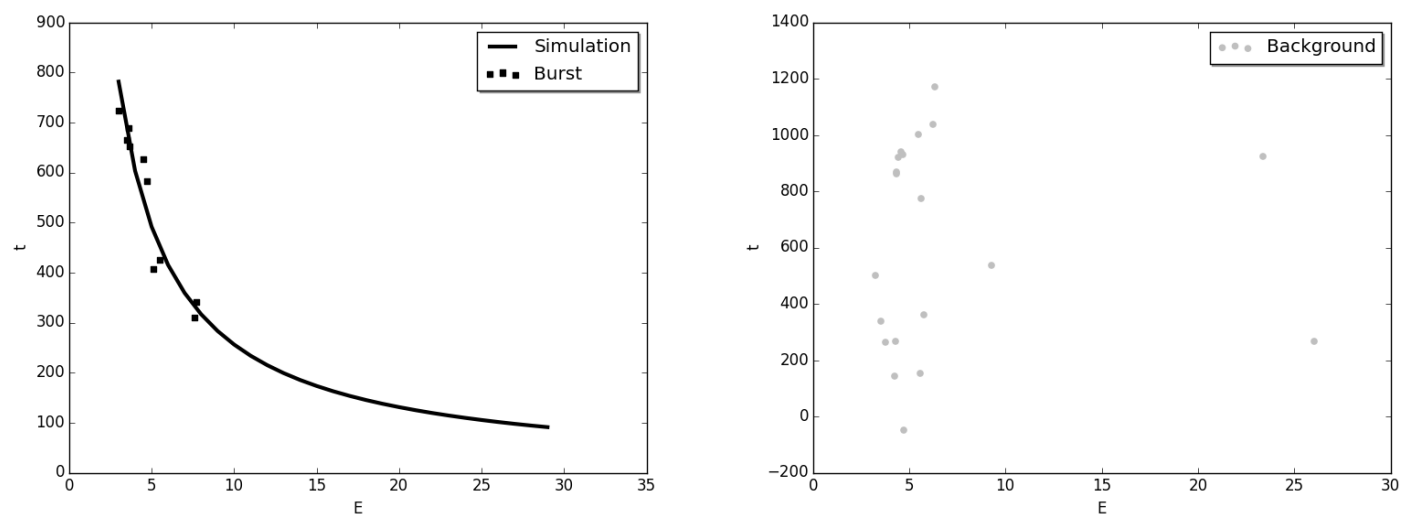

Figure 1: Registered burst electrons (a) and background albedo electrons (b) (Monte-Carlo simulation)

\section{Linear regression method (Ordinary Least Squares)}

$$
R_{\text {linear }}=\sum_{i=1}^{n} \frac{\left(t_{i}-t_{d r}\left(E_{i}, \Delta \lambda\right)\right)^{2}}{\sigma_{i}^{2}}
$$

In real experiment conditions, we can't distinguish burst electrons and background albedo ones, so assume that burst consists of all registered particles, and no background albedo particles. In that case to reconstruct curve and find longitudinal distance between zones of precipitation and registration, a global minimum of ordinary least squares [5] function (3.1) have to be found, where $t_{i}$ - time of particle registration, $E_{i}$ - particle energy, $\sigma_{i}$ - standard deviation of measured particle energy. The result $(\Delta \lambda)$ would be far from reality, because the aforementioned assumption is initially wrong.

\section{Robust regression method}

$$
R_{\text {robust }}=\sum_{i=1}^{n} w_{i} \cdot \frac{\left(t_{i}-t_{d r}\left(E_{i}, \Delta \lambda\right)\right)^{2}}{\sigma_{i}^{2}}
$$

Robust regression analysis is known to be effective in processing data with low signal-to-noise ratio. Main disadvantage of this method is high computation complexity, which can be solved by using of modern computing devices [6]. In order to use robust regression method, one need to weight dataset using some additional information based on assumption that can distinguish "noise" dataset from "signal". The formula of this type of regression is shown on (4.1), where $w_{i}$ weight of particle $i$. 


\section{Minimum linear distance}

As it can be seen from fig. 1a, albedo electrons lays chaotically in relative to the curve, withal burst electrons lay close to the curve. Using this knowledge we assume that the particle lying far from the curve is has less probability to be part of the burst.

$$
\begin{gathered}
d_{i}=\sqrt{\left(E_{i}-E_{\text {min }}\right)^{2}+\left(t_{i}-t_{d r}\left(E_{\text {min }}, \Delta \lambda\right)\right)^{2}} \\
w_{i}=\frac{1}{1+e^{-\left(\beta_{0}+\beta_{1} d_{i}\right)}}
\end{gathered}
$$

In this work we used minimum linear distance from curve to each point (5.1) to measure burst electron likeness using logistic function (5.2) with defined parameters $\beta_{0}$ and $\beta_{1}$, where value $w_{i}=1$, means that particle $i$ - is burst electron, and for value $w_{i}=0$ particle $i$ - is background albedo electron. In this work first approximation curve is calculated using linear regression method, then we calculate $w_{i}$ of particles using their minimum linear distance to first approximation curve. Using robust regression of parameter, one can minimize impact of background albedo electrons on $\Delta \lambda$ calculation. On fig. 2 three curves are drawn: the first approximation curve(linear) drawn in gray, the second approximation curve(robust) drawn in dark gray and the original simulated curve drawn in black. As one can see, linear method failed to converge to original curve, in the same time, robust curve fits almost perfect to it. The results of that exact simulation round are the following: $\Delta \lambda_{\text {linear }}=23 \pm 21$ and $\Delta \lambda_{\text {robust }}=175 \pm 11$ with real $\Delta \lambda_{\text {sim }}=180$.

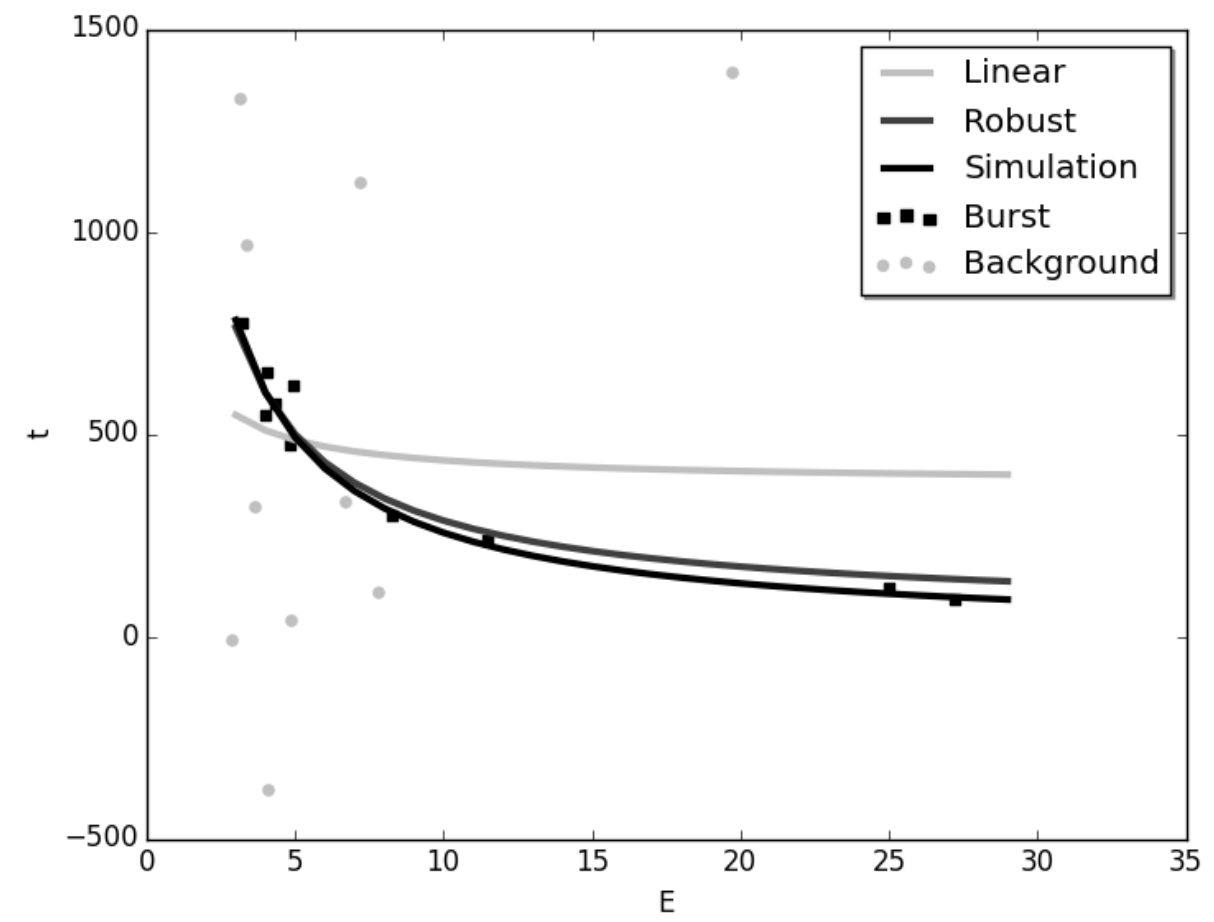

Figure 2: First(linear) and second(robust) curve approximations and orignal(simulated) curve 


\section{Linear and robust regression method comparison}

The study performance study on linear and robust regression method were done to test robust regression method efficiency for various types of bursts. Monte-Carlo method was used to generate burst populations consisting of various number of particles(from ten to one hundred) with albedo electron fluxes from zero to twice of burst particle count.

Figure 3 shows change of average $\Delta \lambda_{\text {err }}=\left|\Delta \lambda_{\text {fit }}-\Delta \lambda_{\text {original }}\right|$ dependence on total electron count in case of equal number of burst and albedo electrons. As it can be seen from the figure, linear regression fails to converge indifferent on total particle count, robust regression converges with some bias because of errors in electron energy identification and incomplete separation of burst and albedo electrons.

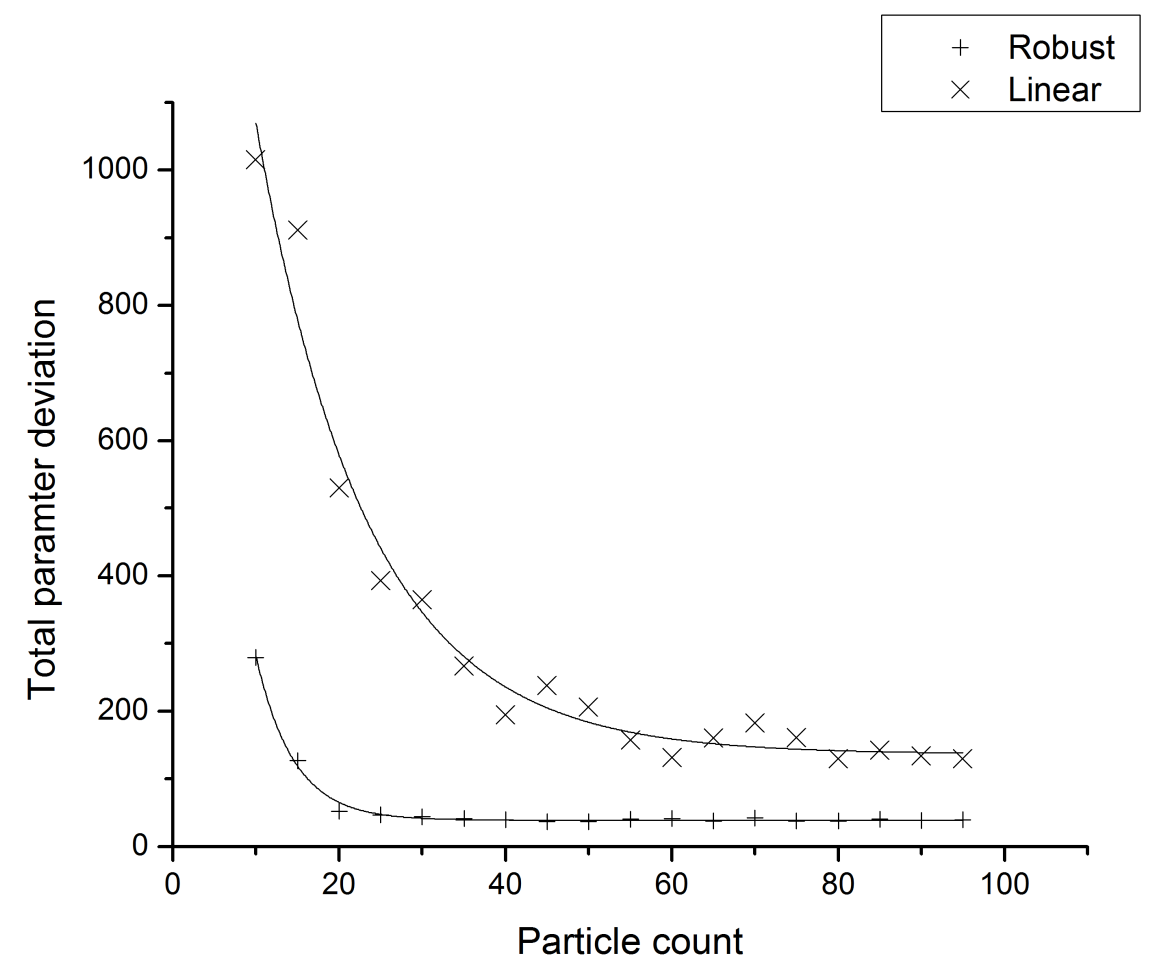

Figure 3: Methods efficiency on the number of registered electrons during the burst

\section{Experimental results}

In this work we applied described above method to burst registered on 24 August 2009(fig. 4). Black dots on this graph are particles which were identified as burst electrons and white dots particles which were identified as albedo electrons. The black curve is the best fit line and gray curves - best fit error borders.

The burst was registered above North Africa region. Using robust fit results $(\Delta \lambda=21 \pm 10)$, we found the estimated location of particle precipitation and it is on the border of African and South American plates. 


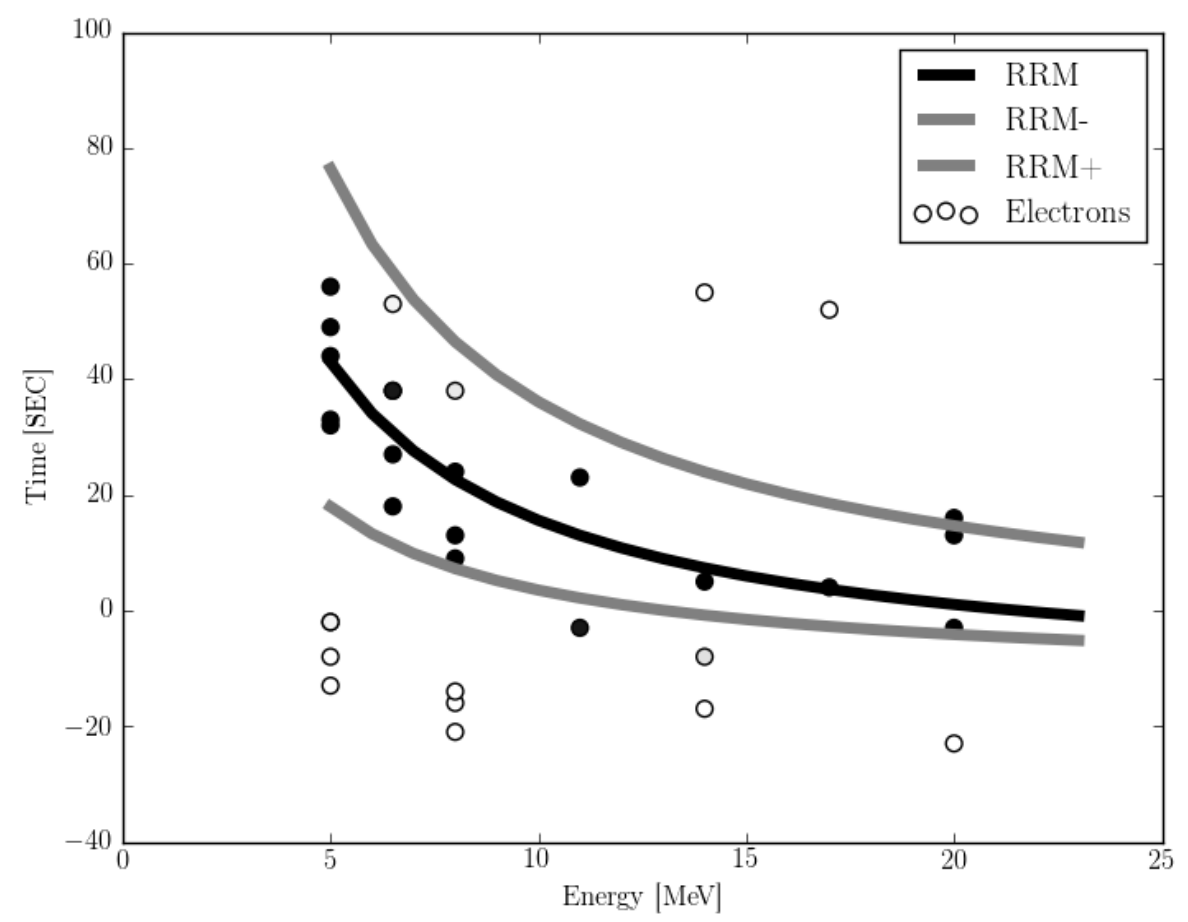

Figure 4: Burst on 24 august 2009 and results of robust regression method

\section{Conclusion}

Using particles energy spectrum during observation time, one can find electron precipitation region relative to point of observation using method of regression analysis. Robust regression analysis proved to be useful on data with high amount of background particles. Proposed method gives biased estimation to longitudinal distance between region of precipitation and point of observation.

\section{References}

[1] Aleksandrin S.Yu, Galper A.M., Voronov S.A. et al. Space and Time Characteristics of High-energy Charged Particles Bursts in Near-Earth Space. // RAS Izvestiya, physical series. 2003 v.67. âDÜ4 pp.79-83.

[2] Aleksandrin S.Yu., Galper A.M., Koldashov S.V., et al. High-energy Charged Particle Bursts in the Near-Earth Space as Earthquake Precursors. // Annales Geophysicae. 2003. V.21. P.597-602.

[3] Aleksandrin S.Yu., Galper A.M., Koldashov S.V. et al. Study of Local Radiation Belt Perturbations in the Satellite Experiments ARINA and VSPLESK. Proceedings of the 31st National Conference on Cosmic Rays, Moscow, Moscow State University, 2010

[4] H. Elliot, The Van Allen Particles, Reports on Progress in Physics, 26, pp.145-180, 1963.

[5] Hastie, T., Tibshirani, R., Friedman, J., The Elements of Statistical Learning, Springer New York Inc., 2001 
[6] Ososkov G., Puzynin I., Polyanskij A. Modern methods of data processing in high energy physics experiments. // Particles and Nucleus, vol.33 (2002), \#3 (russian) 